\title{
PDF Evaluation of the Integer Ambiguity Residuals
}

\author{
S. Verhagen and P.J.G. Teunissen \\ Department of Mathematical Geodesy and Positioning \\ Delft University of Technology, Thijsseweg 11, 2629 JA Delft, The Netherlands \\ E-mail: S.Verhagen@GEO.TUDelft.nl
}

\begin{abstract}
A parameter estimation theory is incomplete if no rigorous measures are available for validating the parameter solution. Since the classical theory of linear estimation does not apply to the integer GPS model, rigorous validation is not possible when use is made of the classical results. As with the classical theory, a first step for being able to validate the integer GPS model is to make use of the residuals and their probabilistic properties. The residuals quantify the inconsistency between data and model, while their probabilistic properties can be used to measure the significance of the inconsistency.

In this contribution we will present and evaluate the joint probability density function (PDF) of the multivariate integer GPS carrier phase ambiguity residuals. Since the residuals and their properties depend on the integer estimation principle used, we will present the PDF of the ambiguity residuals for the whole class of admissible integer estimators. This includes the estimation principles of integer rounding, integer bootstrapping and integer leastsquares. In order to get a better understanding of the various features of the joint PDF of the ambiguity residuals we will use a step-by-step construction aided by graphical means. Although the results apply for any dimension, the one-dimensional case and the two-dimensional case are highlighted.
\end{abstract}

Keywords. GPS, integer ambiguity residuals, parameter distributions

\section{Introduction}

Any GPS model of observation equations that includes carrier phase data of two or more receivers can be parameterized in non-integers and integers. The non-integers refer to the baseline components and additional unknowns like atmospheric delays. The integer parameters refer to the unknown cycle ambiguities of double-differenced carrier phase data. When the integerness of these parameters is explicitily taken into account in the parameter estimation process, we speak of carrier phase ambiguity resolution. It can be applied to a great variety of GPS models that are used in applications like surveying, navigation, and geophysics. An overview of GPS models can be found in textbooks like Hoffmann-Wellenhof et al. (1997), Leick (1995), Parkinson and Spilker (1996), Strang and Borre (1997), Teunissen and Kleusberg (1998).

Parameter estimation provides the estimates of the unknown parameters, together with the corresponding variance matrices. In the classical theory of linear estimation, the variance matrices provide sufficient information on the precision of the estimated parameters. The reason is that a linear model applied to normally (Gaussian) distributed data, provides linear estimators that are also normally distributed, and the peakedness of the multivariate normal distribution is completely captured by the variance matrix.

Unfortunately, this relatively simple approach cannot be applied in case integer parameters are involved in the estimation process, since the integer estimators do not have a Gaussian distribution, even if the model is linear and the data are normally distributed. Instead of the variance matrices, we therefore have to use the parameter distribution itself in order obtain the appropriate measures that can be used to validate the integer parameter solution. For that purpose, the probability density function (PDF) of the ambiguity residuals can be used, since the residuals quantify the inconsistency between data and model, while the PDF describes their probabilistic properties, which are a measure for the significance of the inconsistency.

Our goal is to evaluate the PDF of the ambiguity residuals for the whole class of admissible integer estimators, since the residuals and their probabilistic properties depend on the estimation principle that is used. 
We will start with the formulation of the integer GPS model in section 2, where also the class of admissible estimators is defined. In section 3 the PDF of the ambiguity residuals for the whole class of admissible integer estimators is presented. In practice, the PDF that is commonly used is based on the incorrect assumption that the integer estimator is deterministic. It will be shown how the resulting PDF differs from the correct one as presented here.

The evaluation of the PDFs is the subject of section 4 . We will focus on the one-dimensional and two-dimensional case. Thereby, we will also look at a realistic GPS model. In order to get a good understanding of the various features of the joint PDF of the residuals, it is shown how the PDF can be constructed step-by-step, aided by graphical means. Furthermore, the second moments of the ambiguity residuals with relation to the precision of the GPS data are shown numerically as well as graphically. The PDFs and second moments will be compared to the ones that are usually used in practice.

\section{Integer ambiguity resolution}

Any GPS observation model can be parameterized in integers and non-integers. This gives the following system of linear(ized) observation equations:

$$
y=\underset{m \times n}{A} \underset{n x 1}{a}+\underset{m x p}{B} \underset{p x 1}{b}+\underset{m x 1}{e}
$$

where $y$ is the GPS observation vector of order $m, a$ and $b$ are the unknown parameter vectors of order $n$ and $p$ respectively, and $e$ is the noise vector. The data vector $y$ usually consists of the observedminus-computed DD phase and/or code observations on one, two or three frequencies and accumulated over all observation epochs. The entries of the parameter vector $a$ wil then consist of the unknown integer carrier phase ambiguities, which are expressed in units of cycles rather than in units of range. Since it is known that the entries are integers, $a \in Z^{n}$. The remaining unknown parameters form the entries of the vector $b$. These parameters may be the unknown baseline components and for instance atmospheric (ionospheric, tropospheric) delays, which are all real-valued, i.e. $b \in R^{p}$. In this contribution we will refer to these real-valued parameters as the baseline estimator, although the vector $b$ may thus contain other parameters than only the baseline components.
The classical linear estimation theory can be applied to models that contain real-valued parameters. However, if the integerness of the ambiguity parameters is taken into account, we have to follow a different approach which includes a separate step for ambiguity resolution. The complete estimation process will then consist of three steps (Teunissen 1993). In the first step, the integerness of the vector $a$ is discarded and a 'float' solution is computed with a standard least-squares adjustment. This results in real-valued estimates for $a$ and $b$ and their variance-covariance (vc-) matrix:

$$
\left(\begin{array}{c}
\hat{a} \\
\hat{b}
\end{array}\right), \quad\left(\begin{array}{ll}
Q_{\hat{a}} & Q_{\hat{a} \hat{b}} \\
Q_{\hat{b} \hat{a}} & Q_{\hat{b}}
\end{array}\right)
$$

In the second step the integer ambiguity estimate is computed from the 'float' ambiguity estimate $\hat{a}$ :

$$
\breve{a}=S(\hat{a})
$$

where $S: \quad R^{n} \rightarrow Z^{n}$ the mapping from the $n$ dimensional space of real numbers to the $n$ dimensional space of integers. The final step is to use integer ambiguity estimates to correct the 'float' estimate of $b$ with

$$
\breve{b}=\hat{b}-Q_{\hat{b} \hat{a}} Q_{\hat{a}}^{-1}(\hat{a}-\breve{a})
$$

This solution is referred to as the 'fixed' baseline solution. Both eqs.(2.3) and (2.4) depend on the choice of the integer estimator. Different integer estimators are obtained for different choices of the map $S: R^{n} \rightarrow Z^{n}$. This implies that also the probability distribution of the estimators depends on the choice of the map.

In order to arrive at a class of integer estimators from which to choose, we will start with the map $S$ : $R^{n} \rightarrow Z^{n}$. The space of integers, $Z^{n}$, is of a discrete nature, which implies that the map must be a manyto-one map, and not one-to-one. In other words, different real-valued ambiguity vectors $a$ will be mapped to the same integer vector. Therefore, a subset $S_{z} \subset R^{n}$ can be assigned to each integer vector $z \in Z^{n}$ :

$$
S_{z}=\left\{x \in R^{n} \mid z=S(x)\right\}, z \in Z^{n}
$$

This subset $S_{z}$ contains all real-valued 'float' ambiguity vectors that will be mapped to the same integer vector $z$, and it is called the pull-in region of $z$ (Jonkman 1998, Teunissen 1998). This implies that $\breve{a}=z \Leftrightarrow \hat{a} \in S_{z}$. The integer ambiguity estimator can be expressed as: 


$$
\breve{a}=\sum_{z \in Z^{n}} z s_{z}(\hat{a}) \text { with } s_{z}(x)= \begin{cases}1 & \text { if } x \in S_{z} \\ 0 & \text { otherwise }\end{cases}
$$

where we used the indicator function $s_{z}(x)$.

The integer estimator is completely defined by the pull-in region, so that it is possible to define a class of integer estimators by imposing various conditions on the pull-in regions. The class of admissible integer estimators is defined as follows.

Definition 2.1 (Admissible integer estimators). An integer estimator, $\breve{a}=\sum_{z \in Z^{n}} z s_{z}(\hat{a})$, is said to be admissible when its pull-in region, $S_{z}=\left\{x \in R^{n} \mid z=S(x)\right\}, z \in Z^{n}$, satisfies

$$
\bigcup_{z \in Z^{n}} S_{z}=R^{n}
$$

(ii) Int $S_{z_{1}} \cap \operatorname{Int} S_{z_{2}}=\varnothing, \forall z_{1}, z_{2} \in Z^{n}, z_{1} \neq z_{2}$

(iii) $S_{z}=z+S_{0}, \forall z \in Z^{n}$

where 'Int' denotes the interior of the subset. In Teunissen (1998) the motivation for this definition is given.

Examples of integer estimators that belong to the class of admissible integer estimators are integer rounding $(R)$, integer bootstrapping $(B)$, and integer least-squares $(L S)$. In Teunissen (1999) the corresponding pull-in regions are shown. For a review, see Teunissen (2002b).

\section{The Probability Density Function}

In Teunissen (2002a) the joint and marginal PDFs of both the integer and non-integer parameters were determined. In order to do so, it was assumed that the 'float' solutions are normally distributed. This implies that the marginal PDF of the 'float' ambiguities is given as:

$$
f_{\hat{a}}(x)=\frac{1}{\sqrt{\operatorname{det} Q_{\hat{a}}}(2 \pi)^{\frac{1}{2} n}} \exp \left\{-\frac{1}{2}\|x-a\|_{Q_{\hat{a}}}^{2}\right\}
$$

It can be proven that the joint distribution of $\hat{a}$ and $\breve{a}$ is given by:

$$
f_{\hat{a}, \tilde{a}}(x, z)=f_{\hat{a}}(x) s_{z}(x), x \in R^{n}, z \in Z^{n}
$$

The distributions of the 'float' and 'fixed' ambiguities can be recovered from this PDF by summing over $z$ or integrating over $x$ respectively. The distribution of the integer ambiguity estimator is given by a probability mass function (PMF), and not by a PDF.
This PMF is equal to the integral of the PDF of the 'float' ambiguity over the pull-in region $S_{z}$ :

$$
\int_{R^{n}} f_{\hat{a}, \breve{a}}(x, z) d x=\int_{S_{z}} f_{\hat{a}}(x) d x=P[\breve{a}=z]
$$

Our goal is to determine the PDF of the ambiguity residual, which is defined as:

$$
\breve{e}=\hat{a}-\breve{a}
$$

In practice it is often incorrectly assumed that the integer estimates are deterministic, which would imply that the PDF of the residuals coincides with the PDF of the 'float' ambiguities. Instead, the PDF can be constructed once the joint distribution of $\breve{e}$ and $\breve{a}$ is known. This PDF can be obtained using the transformation (see Teunissen, 2002a):

$$
\left[\begin{array}{c}
\breve{e} \\
\breve{a}
\end{array}\right]=\left[\begin{array}{cc}
I_{n} & -I_{n} \\
0 & I_{n}
\end{array}\right]\left[\begin{array}{l}
\widehat{a} \\
\breve{a}
\end{array}\right]
$$

Since $f_{\hat{a}, \breve{a}}(x)$ is known (eq.(3.2)), the following can be obtained:

$$
f_{\tilde{e}, \tilde{a}}(x, z)=f_{\hat{a}, \breve{a}}(x+z, z)=f_{\hat{a}}(x+z) s_{0}(x)
$$

The PDF of the ambiguity residuals then follows from summing over all integers:

$$
f_{\bar{e}}(x)=\sum_{z \in Z^{n}} f_{\hat{a}}(x+z) s_{0}(x), x \in R^{n}, z \in Z^{n}
$$

With eq.(3.1) this finally gives:

$$
\begin{aligned}
f_{\bar{e}}(x)= & \frac{1}{\sqrt{\operatorname{det} Q_{\vec{a}}}(2 \pi)^{\frac{1}{2} n}} \times \\
& \sum_{z \in Z^{n}} \exp \left\{-\frac{1}{2}\|x-a+z\|_{Q_{\vec{a}}}^{2}\right\} s_{0}(x)
\end{aligned}
$$

Note that $f_{\tilde{e}}(x)$ is only sensitive to the fractional part of $a$ because the summation is over all integers, so that replacing $a$ by $a-[a]$ does not change the result ( $[a]$ means that $a$ is rounded to the nearest integer).

Figure 3.1 shows all steps required for the construction of the PDF of the ambiguity residuals in the one-dimensional (1-D) case. The PDF of $\hat{a}$ (top left) is plotted along the $x$-axis, the PMF of $\breve{a}$ (top right) along the $z$-axis, and the joint PDF (top middle) is plotted in the $x z$-plane. Its construction from the marginal PDF and PMF can be seen as follows. First the parts of the PDF of $\hat{a}$ are sliced out that correspond to all pull-in regions. For the 1$D$ case, the pull-in regions simply are intervals with length 1 , centered at the integers, $S_{z}=\left\{x \in R^{n}|| x-z \mid \leq\right.$ $1 / 2\}$. These slices are then translated along the $z$-axis to the corresponding integers $z$. 

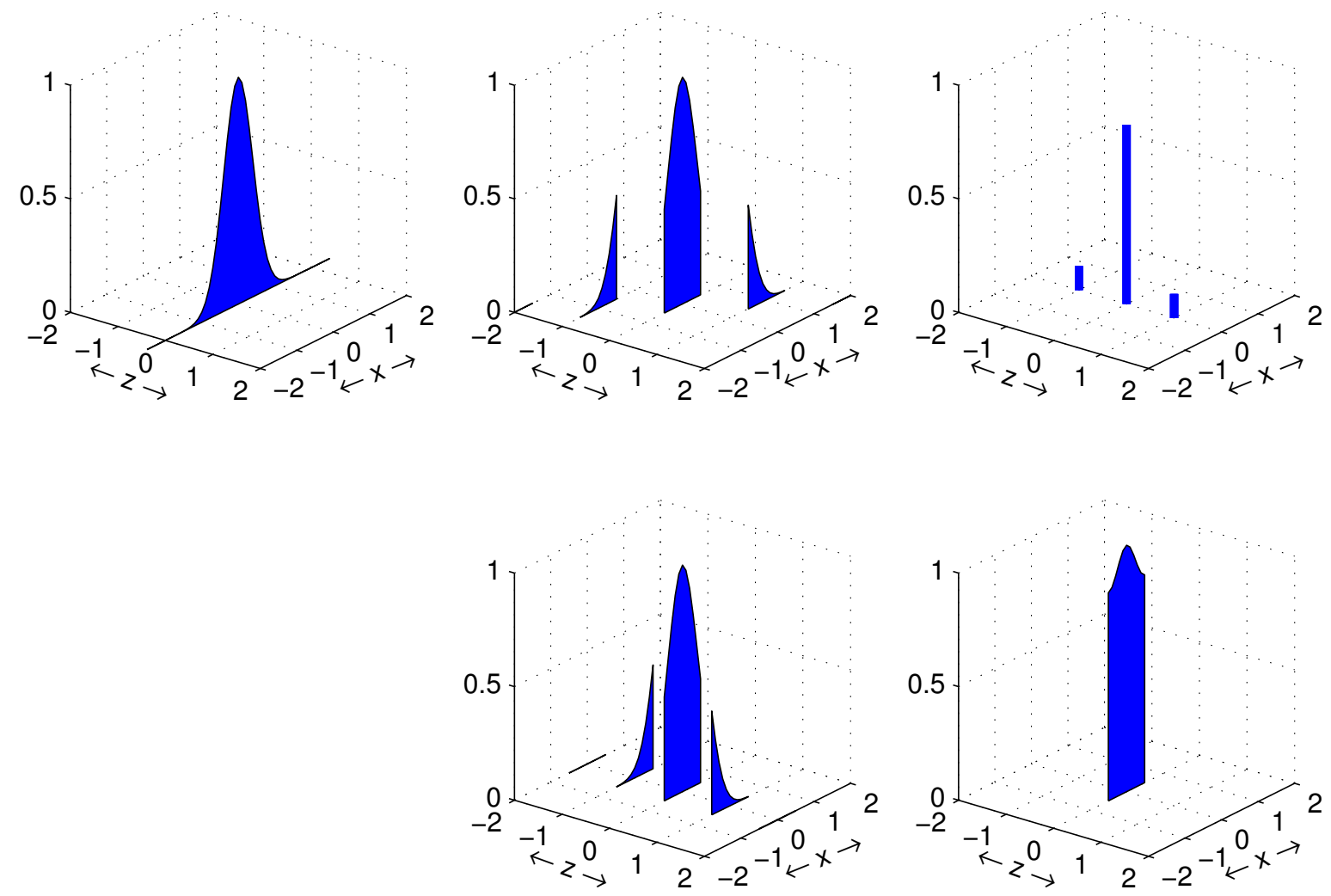

Fig. 3.1. The construction of $f_{\tilde{e}}(x)$ from $f_{\hat{a}}(x)$ : $\operatorname{PDF} f_{\hat{a}}(x)$ (top left); joint $\operatorname{PDF} f_{\hat{a}, \breve{a}}(x, z)$ (top middle); PMF $P[\breve{a}=z]$ (top right); joint $\operatorname{PDF} f_{\check{e}, \check{a}}(x, z)$ (bottom middle); PDF $f_{\tilde{e}}(x)$ (bottom right).

The joint PDF of $\breve{e}$ and $\breve{a}$ (bottom left) follows from another translation of the slices, but now along the $x$-axis, so that they are all centered at the mean value $x=0$. The PDF of $\breve{e}$ (bottom right) is finally obtained by summing over $z$, i.e. all slices are again translated along the $z$-axis to the origin.

The distribution of the ambiguity residuals is clearly non-Gaussian, and it equals zero for all values of $x$ outside the pull-in region. That implies that the norm of the vector of ambiguity residuals is always bounded, irrespective of the value taken by the 'float' solution. A very important implication is that the difference between the 'float' and the 'fixed' baseline solution is then also bounded, as can be seen in eq.(2.4). See also Teunissen (2001).

It can also be seen that the PDF is symmetric around the origin, but the shape depends on the precision of 'float' solution. More examples are given in the next sections. This means that the PDF is independent of the unknown integer ambiguity vector $a \in Z^{n}$. In other words, the mean of the ambiguity residual equals zero, $E\{\breve{e}\}=0$. Thus, the PDF is completely known once the precision of the 'float' solution is known and the choice of the integer estimator is made.

\section{Evaluation of the PDF}

The results of the preceding section apply for any dimension. However, for evaluation we focus on the one-dimensional and two-dimensional case.

\subsection{One-dimensional case}

In the one-dimensional case we do not need to distinguish between the three admissible estimators, since they are all identical. In figure 3.1 a step-bystep graphical construction of the PDF of the residuals was shown. All probability mass is located within the pull-in interval $(-1 / 2,1 / 2)$. The distribution within this interval depends on the precision of the 'float' ambiguities. Figure 4.1 (left panel) shows the PDF of the residuals for different values of the standard deviation, $\sigma$, of the ambiguities. Also, the extremes $\sigma=0$ and $\sigma=\infty$ are shown. In the first case, the PDF becomes an impulse function, in the latter case the residuals have a uniform distribution within the pull-in interval. The peakedness of the PDF clearly depends on the precision. 

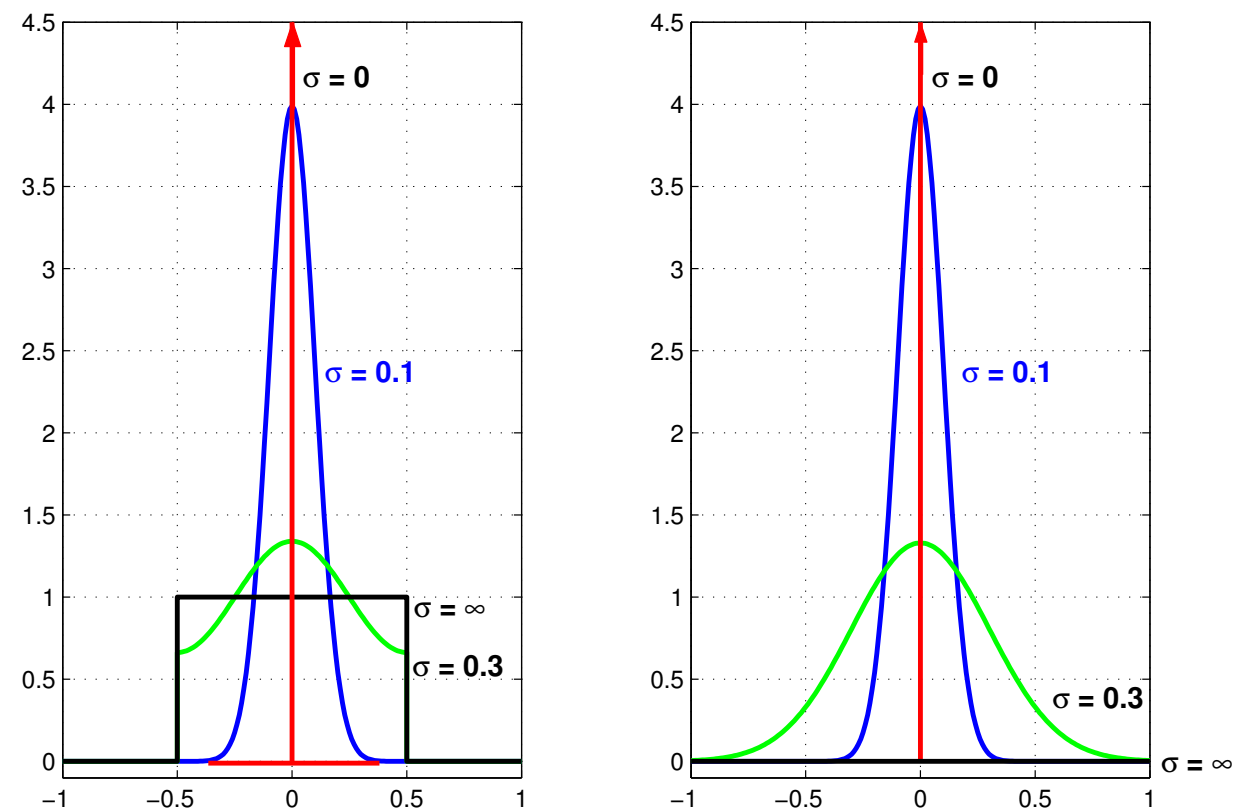

Fig. 4.1. Residual PDF for different values of the standard deviation $\sigma$. correct PDFs (left); and PDFs as used in practice (right).

The right panel of figure 4.1 shows the PDF of the 'float' ambiguities for the same standard deviations. In practice, often the randomness of the 'fixed' solution is ignored, implying that: $f_{\tilde{e}}(x):=f_{\hat{a}}(x)$. This distribution function has infinite tails. Note that when the precision is high, for example $\sigma=0.1$ cycles, that the PDFs become almost identical. On the other hand, when the precision is low, for example $\sigma=0.3$ cycles, the PDFs are very different. If one would you use the false PDF by assuming that the 'fixed' solution is deterministic, that may lead to false hypothesis tests on the integerness of the ambiguities.

Knowledge of the PDF also allows us to derive expressions for the second moments, i.e. the variance and covariance, of the ambiguity residuals. The variance can be computed with:

$$
\sigma_{\breve{e}}^{2}=\int_{x \in S_{0}} x^{2} f_{\breve{e}}(x) d x
$$

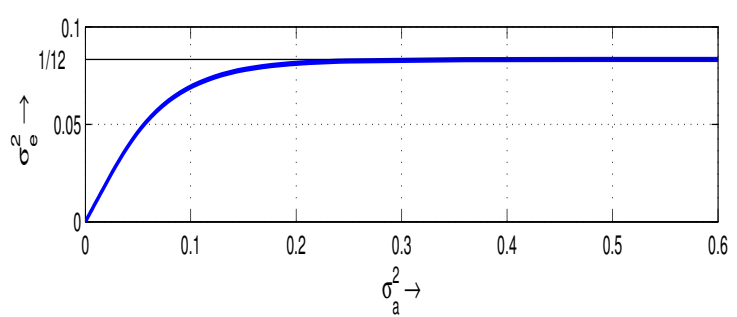

Fig. 4.2. Variance of the residuals, $\sigma_{e}^{2}$, versus the variance of the 'float' ambiguities, $\sigma_{a}^{2},(1-\mathrm{D})$.
Figure 4.2 showns the variance of ambiguity residuals, $\sigma_{e}^{2}$, as function of the variance of the 'float' ambiguities, $\sigma_{a}^{2}$. As can be seen the variance of the residuals is always smaller than $1 / 12$ and it is always lower or equal to the variance of the 'float' ambiguities. Again this shows that it is not correct to assume that the 'fixed' solution is deterministic, so that $\sigma_{\overparen{e}}^{2}:=\sigma_{\widehat{a}}^{2}$

\subsection{Two-dimensional case}

In the two-dimensional case the PDFs of the different admissible estimators may be quite different, especially if there is a high correlation between the 'float' ambiguities. Therefore, we have looked at the resulting PDFs for a vc-matrix that would be obtained by choosing a realistic GPS model. In this example that means that we use the code and phase observations on the L1 and L2 frequency with undifferenced standard deviations of $0.3 \mathrm{~m}$ and $0.003 \mathrm{~m}$ for code and phase observations respectively. The resulting vc-matrix of the DD ambiguities reads:

$$
Q_{\hat{a}}=\left[\begin{array}{ll}
4.9718 & 3.8733 \\
3.8733 & 3.0188
\end{array}\right]
$$

The residual PDF is constructed in a similar way as shown for the 1-D case. This is shown in figure 4.3 in case of rounding: the probability mass of $\hat{a}$ in all pull-in regions, which are squares in this case (top), is added to the mass in the pull-in region centered at 
the origin (middle). The result is the PDF of the residuals shown in the bottom panel (a dark area corresponds to a large probability mass).

Figure 4.4 shows the PDFs of the 'float' ambiguities (top), and of the ambiguity residuals obtained with the different estimators. In figure 4.5 the PDFs of the corresponding 'float' ambiguities and the residuals after $Z$-transformation are shown. This $Z$-transformation is used to decorrelate the vcmatrix (see e.g. Teunissen, 1998):

$$
Q_{\hat{z}}=Z^{T} Q_{\hat{a}} Z, \hat{z}=Z^{T} \hat{a}
$$

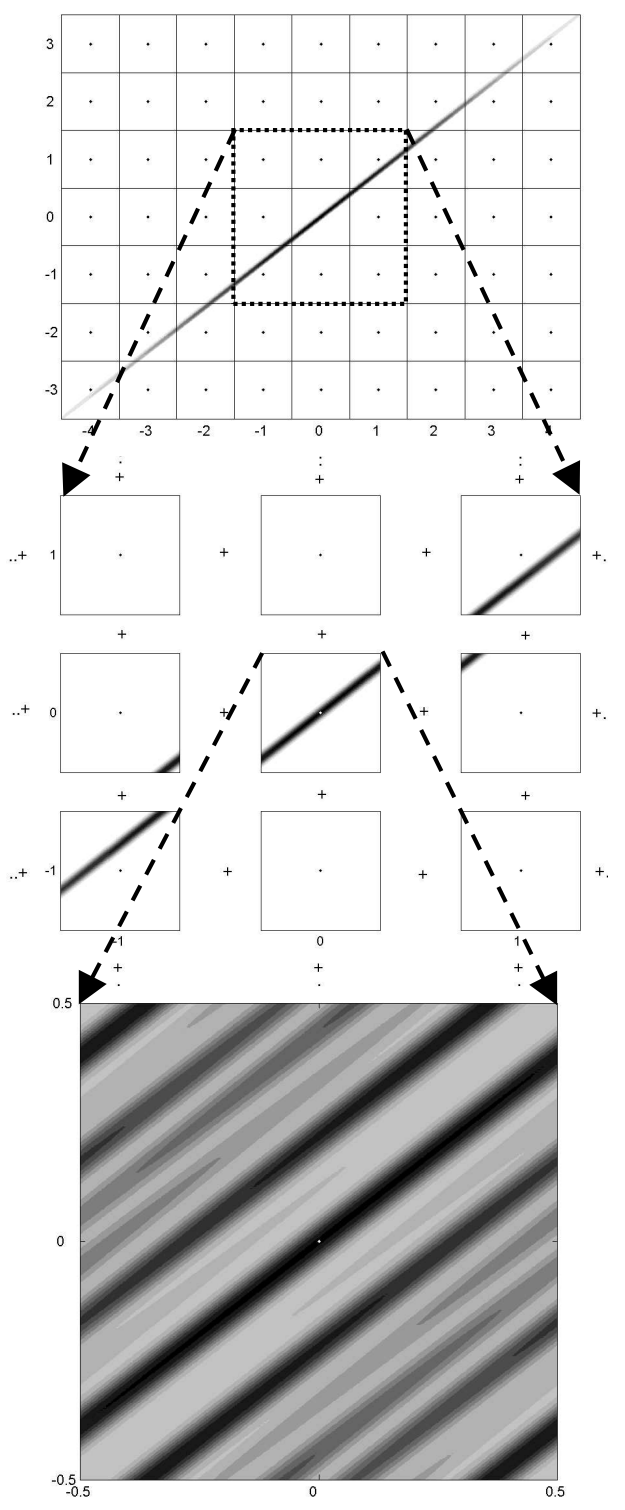

Fig. 4.3. The construction of $f_{\tilde{e}}(x)$ from $f_{\hat{a}}(x)$ in case of rounding (2-D): PDF $f_{\hat{a}}(x)$ (top); summation over all pull-in regions (middle); $\operatorname{PDF} f_{\tilde{e}}(x)$ (bottom).
In our example this results in:

$$
Q_{\hat{\imath}}=\left(\begin{array}{ll}
0.0865 & 0.0364 \\
0.0364 & 0.0847
\end{array}\right), Z=\left(\begin{array}{rr}
-3 & -4 \\
4 & 5
\end{array}\right)
$$

If we first look at the results obtained for the vcmatrix given in (4.2) we see that the distribution clearly depends on the integer estimator that is used, and that it may be considerably different from the PDF of $\hat{a}$. Firstly, the PDF of $\hat{a}$ is not bounded, whereas the residual PDFs are bounded by the corresponding pull-in region. Secondly, the residual PDFs in this example are multimodal. This is due to the fact that the PDF of $\hat{a}$ is very elongated and its orientation is not along the $x_{1^{-}}, x_{2^{-}}$or $x_{1}=x_{2}$-axis. This implies that there is also a high probability mass located far away from the origin. So, this confirms that the 'fixed' ambiguities may not always be considered deterministic.

The PDF of the residual for integer LS, $\breve{e}_{L S}$, is shown in the bottom panel of figure 4.4. The pull-in region of the integer LS estimator follows very much the shape of the PDF of $\hat{a}$ and is thus very elongated. Therefore, it is hard to visually detect any differences between the PDFs of $\hat{a}$ and $\breve{e}_{L S}$, although there are differences especially near the boundaries of the pull-in region and of course outside the pull-in region. However, this will be easier to see for the residuals of the $Z$-transformed ambiguities. Note that only for the integer LS estimator the following is true:

$$
\breve{e}_{z, L S}=\hat{z}-\breve{z}_{L S}=Z^{T}\left(\hat{a}-\breve{a}_{L S}\right)=Z^{T} \breve{e}_{L S}
$$

so that

$$
f_{\bar{e}_{2, L S}}(x)=f_{\tilde{e}_{L S}}\left(Z^{-T} x\right)
$$

Therefore, the shape of the PDF of the Ztransformed residuals is similar to the PDF of the untransformed residuals, only that it is transformed like the pull-in region.

Figure 4.5 shows that especially for the bootstrapped and integer LS estimator the shape of the residual PDF 'fits' the shape of the pull-in region quite well. Due to the decorrelation, the resulting PDFs are now unimodal. However, the shape near the boundaries of the pull-in regions is clearly different from the shape of the PDF of $\hat{a}$. For clarity this is shown by the black contour lines which are plotted at a value of 0.6. Note that these contour lines are not closed, which means that biases in certain directions will be harder to detect than biases in other directions, depending on the correlation between the 'float' ambiguities. 

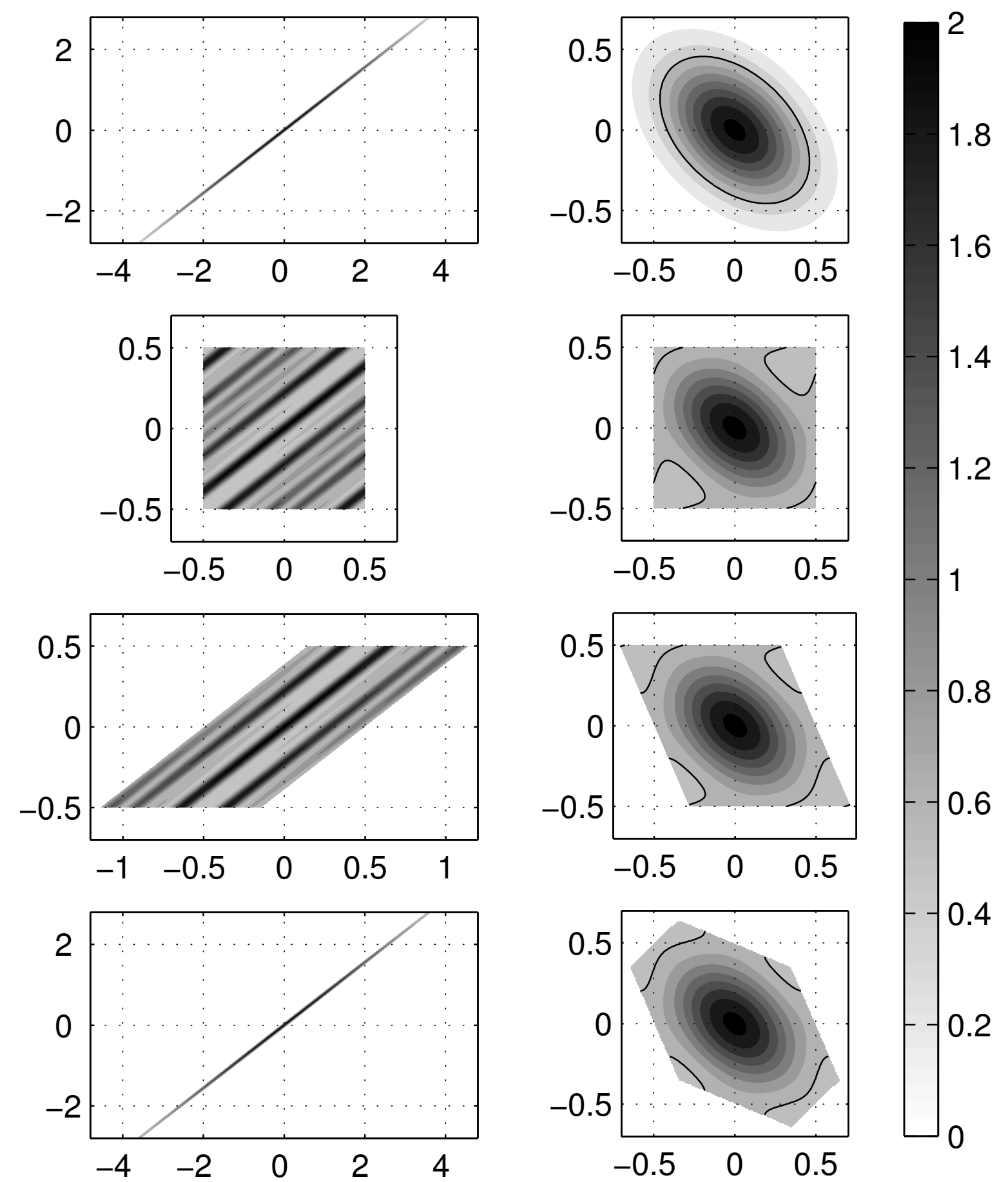

Fig. 4.4. Residual PDFs (2-D): $\operatorname{PDF} f_{\hat{a}}(x)$ (top); $\operatorname{PDF} f_{\tilde{e}}(x)$ for rounding ( $2^{\text {nd }}$ from top); PDF $f_{\tilde{e}}(x)$ for bootstrapping $\left(3^{\text {rd }}\right.$ from top); PDF $f_{\tilde{e}}(x)$ for integer LS (bottom).

Fig. 4.5. Residual PDFs after Z-transformation (2-D): PDF $f_{\hat{a}}(x)$ (top); PDF $f_{\tilde{e}}(x)$ for rounding ( $2^{\text {nd }}$ from top); PDF $f_{\tilde{e}}(x)$ for bootstrapping ( $3^{\text {rd }}$ from top); PDF $f_{\tilde{e}}(x)$ for integer LS (bottom). 
Although the PDFs of $\hat{a}$ and $\breve{e}_{z, L S}$ have the most similar shapes, the figure shows that the distributions are different from each other. Again the conclusion is that ignoring the randomness of the 'fixed' solution leads to false assumptions on the distribution and thus to false tests for integerness of the parameters.

The second moments of the ambiguity residuals in case of rounding are computed with:

$$
\sigma_{\breve{e}_{i} \breve{e}_{j}}=\iint_{x \in S_{0}} x_{i} x_{j} f_{\check{e}_{i} \breve{e}_{j}}\left(x_{i}, x_{j}\right) d x_{i} d x_{j}
$$

The resulting vc-matrices are:

$$
\begin{aligned}
Q_{\breve{e}_{R}} & =\left(\begin{array}{rr}
0.0833 & -0.0007 \\
-0.0007 & 0.0833
\end{array}\right) \\
Q_{\check{e}_{z, R}} & =\left(\begin{array}{rr}
0.0650 & -0.0067 \\
-0.0067 & 0.0643
\end{array}\right)
\end{aligned}
$$

Figure 4.6 shows the ellipses that correspond to these vc-matrices of the residuals without and with $Z$-transformation. Also, the corresponding vcmatrices of the 'float' ambiguities, $Q_{\hat{a}}$ and $Q_{\hat{z}}$, are shown. These are the second moments of the residuals that are usually used in practice. It can be seen that especially for the highly correlated vcmatrix, the deviation is large.

\section{Conclusions}

In this contribution it is shown how the PDFs of ambiguity residuals can be constructed. This allows for rigorous testing. The next step will be to formulate hypotheses and determine test statistics sensitive to these hypotheses for the $n$-dimensional case for all admissible integer estimators. Thereby we will have to look at the size and the power of the test and the test should be optimal.

Currently, the randomness of the 'fixed' solution is ignored, which results in wrong residual PDFs, and wrong second moments. This may lead to a false evaluation of the quality of testing the integerness of the ambiguities. The knowledge of the correct PDFs can be used to investigate in which cases the test as used in practice gives a sufficient description, and in which cases it will lead to false conclusions.
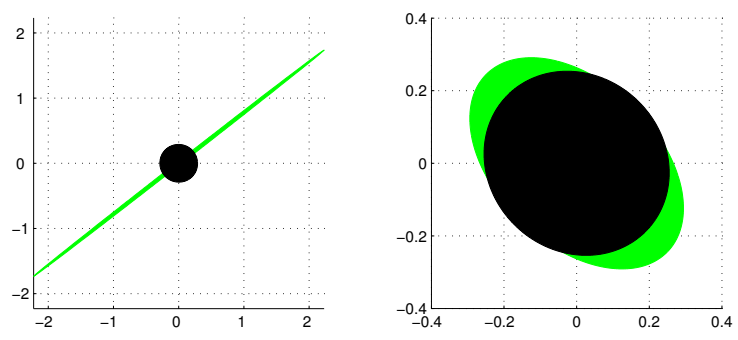

Fig. 4.6. Ellipses corresponding to the vc-matrices of the 'float' ambiguites (light grey) and the residuals (black): for $Q_{\hat{a}}$ (left) and $Q_{\hat{z}}$ (right).

\section{References}

Hoffmann-Wellenhof, B., H. Lichtenegger, and J. Collins (1997). Global Positioning System: Theory and Practice. $4^{\text {th }}$ edn., Springer, Berlin Heidelberg New York.

Jonkman, N.F. (1998). Integer Ambiguity Estimation without the Receiver-Satellite Geometry. LGR Series, no.18, Delft Geodetic Computing Centre, Delft.

Leick, A. (1995). GPS Satellite Surveying. $2^{\text {nd }}$ edn., John Wiley, New York.

Parkinson, B. and J.J. Spilker (eds) (1996). GPS: Theory and Applications. Vols. 1 and 2, AIAA, Washington, DC.

Strang, G. and K. Borre (1997). Linear Algebra, Geodesy, and GPS. Wellesley-Cambridge Press.

Teunissen, P.J.G. (1993). Least-squares Estimation of the Integer GPS Ambiguities. Invited lecture, Section IV Theory and Methodology, IAG General Meeting. Beijing, August. Also in: $L G R$ Series, No.6, Delft Geodetic Computing Centre, Delft, pp. 59-74.

Teunissen, P.J.G. (1998). On the Integer Normal Distribution of the GPS Ambiguities. Artific Sat 33(2): 49-64.

Teunissen, P.J.G. (1999). An Optimality Property of the Integer Least-squares Estimator. J Geod 73: 587-593.

Teunissen, P.J.G. (2001). Integer Estimation in the Presence of Biases. J Geod 75: 399-407.

Teunissen, P.J.G. (2002a). The Parameter Distributions of the Integer GPS Model. J Geod 76: 41-48.

Teunissen, P.J.G. (2002b). Integer Least-Squares. In: Proc. V Hotine-Marussi Symposium. Matera, June 17-21 (this volume).

Teunissen, P.J.G., and A. Kleusberg (eds) (1998). GPS for Geodesy. $2^{\text {nd }}$ edn., Springer, Berlin Heidelberg New York. 